\title{
Modelling Fleet Performance over Complex Operating Scenarios
}

\author{
Iain Davison* MSc, BSc, MMath, MIMA, MSaRS \\ * Principal Consultant, Atkins \\ * Corresponding Author. Email: Iain.Davison@Atkinsglobal.com
}

\begin{abstract}
Synopsis
The Systems Availability Model (SAM) is a program designed to assess the Availability, Reliability and Maintainability (AR\&M) characteristics of multiple systems used over operating scenarios that place varying demands upon those systems, such as that encountered in complex military, commercial shipping, industrial installations and deployed systems of systems.

The unique ability of SAM to overlay system dependencies onto complex mission profiles makes it a uniquely powerful and flexible AR\&M modelling tool. Mission profiles are built up from a variety of activities, each demanding use of different combinations of equipment, rather than a fixed time at risk approach adopted by many simpler modelling tools.

This paper and associated presentation discusses:

- The unique capabilities of SAM and, at a high level, how a SAM model is developed and its crossindustry applications;

- The use of SAM to set system/equipment requirements, and understand the impact of equipment reliability on a fleet of ships undergoing complex operating scenarios;

- Reflecting changes to mission requirements, and the knock-on effect of predicted performance;

- How SAM can be used to understand the significance of individual systems during safety critical activities (e.g. replenishment at sea, close water navigation).
\end{abstract}

Keywords: Availability; Reliability; Maintainability; Modelling; Asset Management.

\section{Introduction}

The purpose of this paper is to discuss the role of Availability, Reliability and Maintainability (AR\&M) modelling through the life cycle of complex systems or fleets of equipment. Specifically the paper considers the good practice of modelling of multiple systems used over operating scenarios that place varying demands upon those systems, such as that encountered in complex military, commercial shipping, industrial installations and deployed systems of systems.

The paper discusses the role of modelling in setting of equipment end-user/system/equipment requirements, and understanding the impact of equipment reliability on a complex system or fleet undergoing complex operating scenarios and how the features of the Systems Availability Model (SAM) lend it to support such activities. The role of modelling in the subsequent management of project risk is then discussed with emphasis on two key areas:

- The importance of reflecting changes to mission requirements, and the knock-on effect of predicted performance; and

- The importance of understanding the significance of individual functions or sub-systems (e.g. during safety critical activities such as replenishment at sea, close water navigation).

A final section to the paper is provided that summarises the unique capabilities of SAM that allow it to support the above and, at a high level, how a SAM model is developed and its cross-industry applications.

Although this paper refers to methodology specifically provided by SAM, it should be noted that other commercially available tools can be employed to provide similar methodology and benefits.

\footnotetext{
Author's Biography

Iain Davison is a Principal Consultant at Atkins in Aldershot, UK. Iain is a Chartered Mathematician with over 12 years' experience in Availability, Reliability and Maintainability modelling of complex systems or systems, and Safety Engineering (particularly in Air Traffic Management and Nuclear domains).
} 


\section{What is SAM?}

The SAM is a program designed to assess the AR\&M characteristics of multiple systems used over operating scenarios that place varying demands upon those systems, such as that encountered in complex military, industrial installations and deployed systems of systems.

The unique ability of SAM to overlay system dependencies onto complex mission profiles makes it a powerful and flexible AR\&M modelling tool. Mission profiles are built up from a variety of activities, each demanding use of different combinations of equipment, rather than a fixed time at risk approach adopted by many simpler modelling tools.

SAM is a UK Ministry of Defence (MOD) owned tool, managed and supported by Atkins, issued without charge to UK MOD Projects, and is commercially available to anyone else.

SAM is designed to provide AR\&M predictions for single systems or groups of interdependent systems operating over a mission.

SAM was initially developed for the MOD to depict the interactions between a ship's systems (weapons, power distribution, sensors, propulsion, etc.) in the various roles a ship may be required to undertake. For example, Figure 1 presents a representation of how multiple power requirements for a ship can be modelled in SAM. However, successful developments have extended the capabilities of SAM and it is now capable of modelling any system, process plant or engineering installation.

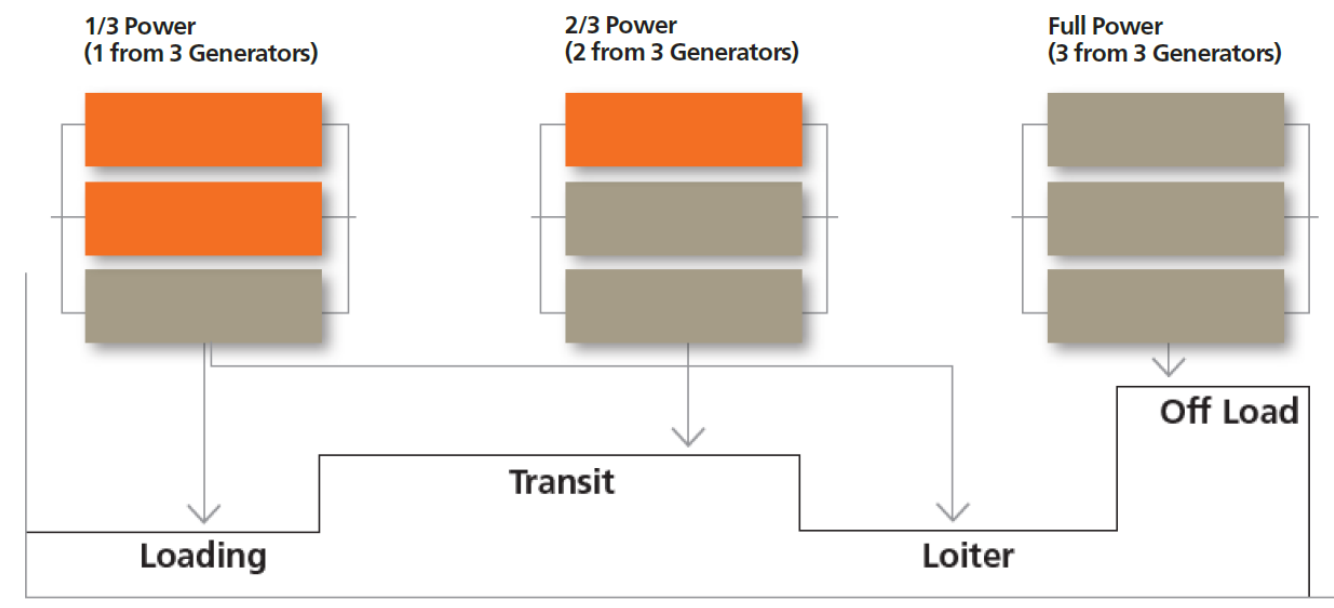

Figure 1: Modelling Multiple Power Requirements

SAM is designed to be a flexible program capable of assessing a variety of systems (including interdependent systems) of mixed repairable and non-repairable equipment, including those with active or standby redundancy. It includes the capability to model first line (local) and second line logistic delays and maintainer availability.

\section{Setting of Requirements}

\subsection{Introduction}

As described in the Part B Chapter 2 of the Applied R\&M Manual for Defence Systems (referred to as GR-77) [1], the achievement of AR\&M in service stems from either luck or a clear, appropriate and realistic specification of the requirements together with a contract that enforces adherence.

The Safety and Reliability Society (SaRS) aims to provide an evolving body of knowledge for professionals. GR77 is a web-based resource in the body of knowledge that provides technical AR\&M guidance on a wide range of topics under the headings of "General Guidance", "R\&M Related Activities", "R\&M Related Techniques", "Supporting Theory", etc.

AR\&M requirements and their traceability to definitions, assumptions and lower level requirements or constraints can be problematic, particularly where there is significant change in the technical requirements or frequent changes in project personnel. This lack of traceability can result in shortcomings in the AR\&M requirements and contractual requirements, which in turn may compromise capability and escalate through life costs.

Part B Chapter 2 of GR-77 [1], provides guidance on the methods available to setting AR\&M requirements and maintaining their traceability. In particular, where equipment end-user requirements are satisfied by a number 
of system level requirements, it is not always clear to the equipment end-user which of the many AR\&M related attributes are required at the concept stage of a project. GR-77 [1] advocates the use of scenario analysis supported by AR\&M modelling or analysis to help identify AR\&M attributes critical to mission success. The subsequent sections describe how AR\&M modelling tools and techniques may be used to aid the development of equipment end-user requirements, apportionment to system level requirements, and demonstrating the linkage between them.

\subsection{Equipment End-User Requirements}

\subsubsection{Initial Model Creation}

As described in Appendix A (Section 6), a SAM model is built from the bottom up (i.e. beginning with defining equipment/components, then moving on major functions and a mission profile). However, since equipment enduser requirements are defined at a high level (e.g. availability of a fleet of assets), when developing a model to define or validate the requirements it can be useful to initially apply a more top-down approach.

Through engagement with equipment end-users and other key stakeholders, the mission profile for a fleet of assets should be developed. Initially, this may simply be expressed as general periods of "Activity" and "Nonactivity", for each asset. Through continued engagement, the profile should be developed to include more specific activities (typically categorised as mission essential, critical and non-critical events), which can eventually be linked to the major functions performed by each asset.

A useful way of visually representing this linkage between activity and major function is through a criticality matrix. It is recommended that the classification descriptors used in a criticality matrix should be developed on a project-by-project basis to allow specific project nuances to be considered. Figure 2 shows an example criticality matrix employing the following descriptors:

- Desirable (D), i.e. the function is desired for the mission, but is not required for mission success;

- Mission (M), i.e. the function is required for mission success;

- Critical (C), i.e. the function is critical to both mission success and the wider asset performance (potentially safety related).

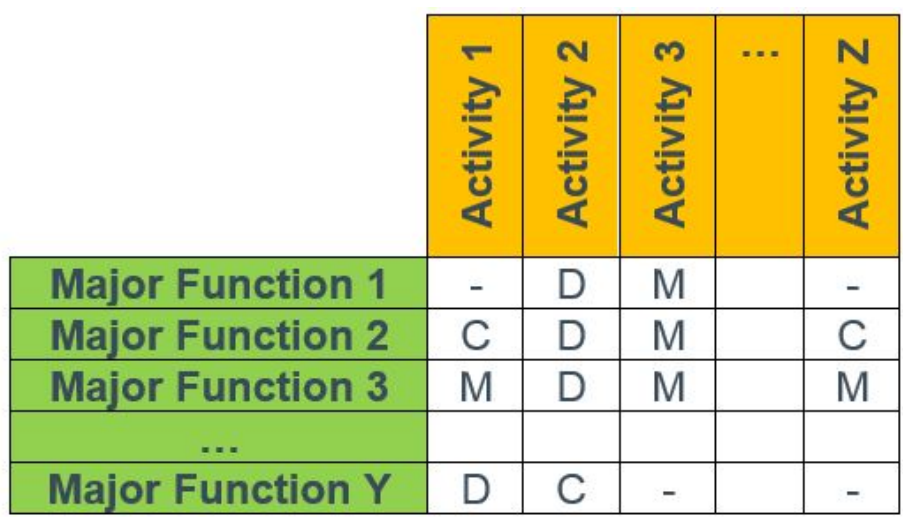

Figure 2: Example Criticality Matrix (Major Function v Activity)

This criticality matrix is intended to show the direct link between the major functions and activities. In reality, further subtleties may exist in relation to reversionary modes of operation and the assessment of criticality (D, M or C). For example, "Major Function 1" may be achieved by three different modes of operation, represented by the reliability block diagrams shown in Figure 3. As such, the criticality matrix may be further developed to articulate how critical each mode of operation is for each activity, as shown in Figure 4. 


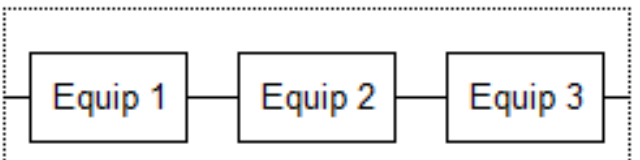

Full Operating Capability
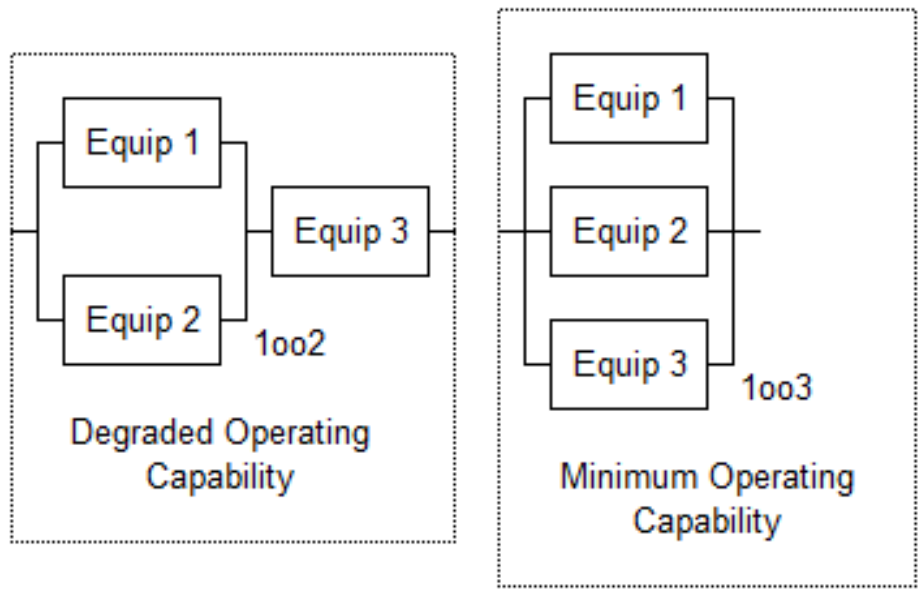

Figure 3: Example Modes of Operation

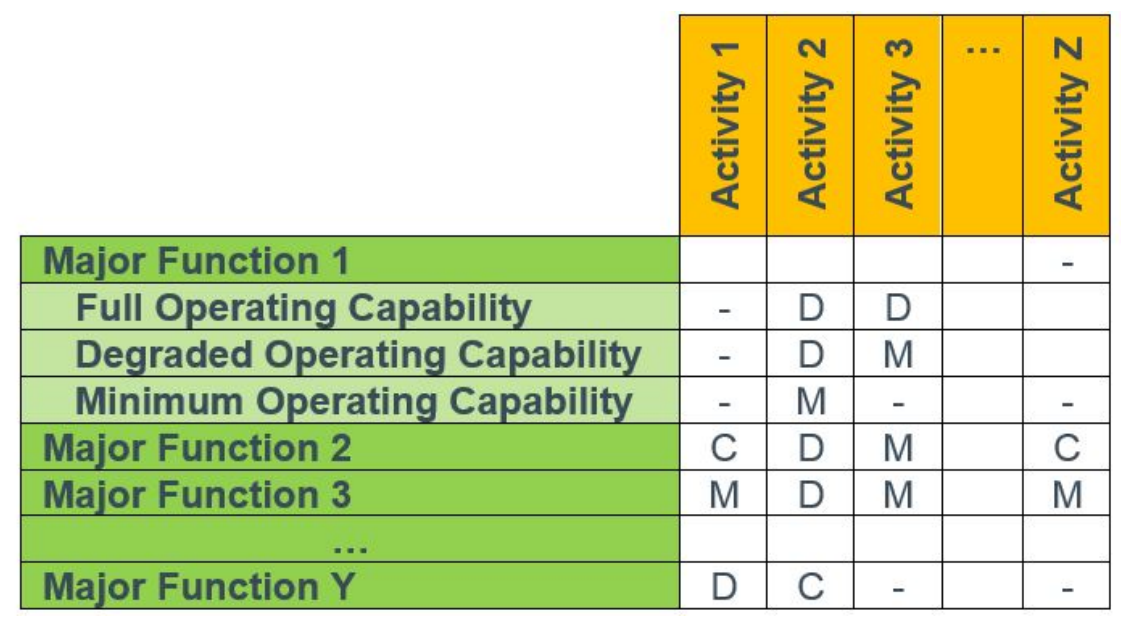

Figure 4: Example Criticality Matrix (Modes of Operation v Activity)

The key systems and sub-systems required to perform each major function can then be determined and defined at a level appropriate to the stage of the project. For example, during concept phase, details of an asset may only be described at a high level, such as "Propulsion", "Communications", etc.

Once these attributes have been determined, a SAM model can be created and populated in the traditional sense, from the bottom up (also see Appendix A (Section 6) for details of how to construct a SAM model):

- Key systems and sub-systems populated into a SAM Model Equipment List (MEL); initially with dummy reliability and maintainability data, to be populated later;

- $\quad$ Functions built up of the key systems and sub-systems into a SAM Availability Dependency Chart (ADC);

- Activities built up from functions and formed into mission profile(s) into a SAM Mission Profile Chart (MPC).

\subsubsection{Development of Reliability and Maintainability Model Data}

The generation and sourcing of reliability and maintainability data of suitable provenance is generally one of the more challenging activities in the creation of an AR\&M model. Since details of the asset are likely to be limited at the early stages of a project, the best source of reliability and maintainability data is expected to be from the inservice experience of existing similar assets. Furthermore, a common equipment end-user request is that a new asset is no less reliable than an existing asset; understanding the performance of the existing asset is therefore key to defining equipment end-user requirements.

In an ideal world, this in-service performance would be based on fully traceable data including details of usage, environment and from a statistically significant sample size over a number of years, which can be fully 
interrogated. However in reality, this data may be patchy at best, and as such the experience of the equipment endusers should be used to validate any reliability and maintainability data gleaned. It is important for the validation exercise that data is presented in a form that equipment end-users can relate to and can understand. For example, rather than presenting the reliability of an asset function as a Mean Time Between Failure (MTBF) or failure rate per million hours, the equipment end-user may relate better to an expected number of failures per annum or failures per mission.

Once reliability and maintainability data for each key system or sub-system is developed, it can be populated into the SAM model. In order to articulate an equipment end-user requirement, it may also be necessary to reflect the expected support structure into the AR\&M metrics. In the concept phase, this may be represented more simply as a probability of spare being available, and a logistic delay time.

As shown in Figure 6, SAM is capable of providing a number of outputs and metrics including availability, which is considered the most appropriate for defining equipment end-user requirements.

\subsubsection{Model Sensitivity Analysis}

Arguably, the main benefit in employing a modelling tool, such as SAM, to define requirements is the ability to perform sensitivity and "what if" analysis. This allows the equipment end-user to gain an understanding of the impact of decisions on the overall AR\&M performance of an asset and hence where areas of risk reside. In particular, during the early project phases it may be common to assess the impact of changes in (where applicable) fleet size, asset functionality and mission profile, on modelling results.

Before undertaking sensitivity analysis, it may be beneficial to present the initial modelling results to the equipment end-users and all stakeholders. This allows a level of validation and managing of expectations against "gut-feel". At this point it is also advisable to highlight any particular areas of uncertainty and the modelling features (e.g. system reliability, asset architecture, mission profile, etc.) driving the initial results. The various outputs of SAM, as discussed in Appendix A (Section 6), can be used by the modeller to investigate the drivers of unreliability/unavailability.

The particular areas to be assessed in sensitivity and "what if" analysis should be discussed and agreed with the equipment end-user and stakeholders, based on the areas of risk and uncertainty. It is useful to document this in the form of a plan, particularly where multiple parameters are requested to be investigated, to ensure a logical and structured approach is maintained. Results from each sensitivity case can be presented together with the baseline initial results and discussed with the equipment end-user, such that they get an appreciation of how various decisions can influence the AR\&M metrics.

\subsubsection{Setting Equipment End-User Requirements}

Results from the SAM modelling activity can be used to set or validate equipment end-user AR\&M requirements for a project.

If the equipment end-user requirements have already been defined (for example derived from an existing asset), comparing these requirements with results from the SAM model gives a measure of confidence that the metrics are realistic and achievable, based on the model inputs provided.

Differences ${ }^{1}$ between the SAM model results and defined equipment end-user requirements should be investigated to understand the reasons and their significance. If the difference is relatively small and can be reduced by slight adjustments to the model input data (e.g. system/sub-system reliability and maintainability data, or mission profile), little or no change to the equipment end-user requirements is probably necessary. However, the nature of the difference should be discussed and agreed with the equipment end-user and stakeholders.

If there is a more significant difference between the SAM modelling results and equipment end-user requirements, the basis of equipment end-user requirements should be compared with the basis of the SAM model to understand if there are any fundamental reasons why the results should differ. If the two are based on similar foundations, further interrogation of the model will be required to gain an understanding of the source of the difference. Once established, the reason(s) for the significant difference should be discussed with the equipment end-user and stakeholders to agree whether changes to the SAM model are required, and whether the SAM modelling results should be used to update the equipment end-user AR\&M requirements. Of course, it may be the case that the equipment end-user requirements remain unchanged due to a higher-level operational requirement. If this is the case, then the SAM model can be used to derive system requirements necessary to achieve the equipment end-user requirements, as discussed in Section 3.3.

\footnotetext{
${ }^{1}$ Due to inherent uncertainty in reliability data, and the Monte Carlo simulation techniques employed by SAM, it is considered highly unlikely that the SAM model results will match the derived equipment end-user requirements exactly - unless, of course, the SAM model results have been used to derive the requirements.
} 
Where the equipment end-user requirements have yet to be defined, the results from the SAM model can be employed to do so, providing confidence that, based on the given input, the metrics are realistic and achievable.

In both cases (setting or validating equipment end-user requirements), sensitivity analysis is an important activity, providing the equipment end-user with an appreciation of the limitations of the equipment end-user requirements and how changes to the model inputs can affect the AR\&M metrics. This should be taken into consideration when defining the equipment end-user requirements, particularly in areas where there remains significant uncertainty (e.g. asset functions, or mission profile).

\subsection{System Requirements}

Once a SAM model has been created to either set or validate the equipment end-user AR\&M requirements it can be used directly to derive system requirements. As the SAM model has been built up from key functions, systems and sub-systems, the AR\&M metrics for each can be extracted directly from the SAM model results and used to form the system requirements.

The advantage of this approach is that it provides a clear linkage between equipment end-user and system requirements; meeting system requirements ensures that the equipment end-user requirements are also achieved.

The level at which the system requirements are presented is likely to depend on contractual arrangements. For example, if it is expected that a single supplier is responsible for the complete asset, the system requirements may be presented at a relatively high level, leaving the supplier responsible for further apportioning these to lowerlevel sub-system suppliers. If, however, it is expected that the project will be contracted to multiple suppliers, the level at which the system requirements are articulated should reflect this.

Linked with the development of system requirements is the opportunity to set up the contracted method for monitoring in-service AR\&M performance and, in particular, Key Performance Indicators (KPI) and metrics specific to the project, which can be developed from and assessed against the SAM model. GR-77 Part B Chapter 2 [1] discusses various types of AR\&M metrics, which may be employed when setting system requirements and KPIs. The pros and cons of the various metrics should be discussed with the equipment end-user and stakeholders to determine the most appropriate AR\&M metrics to be used. Once decided upon, SAM's flexibility allows the model results to be presented in the required form, for use in setting system requirements. Metrics that can be derived from a SAM model include (but are not limited to):

- Availability - Operational or intrinsic availability (of a fleet, individual assets, systems, sub-systems, etc.).

- $\quad$ Probability of failure over a given period - Reliability; Basic/logistic reliability; Non-repairable at sea reliability; Mission reliability.

- Rate of failures of period between failures - Mean variate ${ }^{2}$ between failures; Mean variate between mission failures; Characteristic life.

- $\quad$ Maintenance performance - Active Repair Time (ART); ART per operating hour; Mean Time To Repair (MTTR); False alarm rate; Total corrective maintenance time.

- Cost of ownership ${ }^{3}$ - Labour; Spares and materials costs associated with failures.

- Others - Failure free operating period.

\subsection{Supporting the Initial $R \& M$ Case}

Def Stan 00-42 Part 3 [2] and BS EN 62741 [3] provide a description of the principles of progressive assurance, with the former reflecting specifically the current policy within UK defence acquisition. Both standards provide guidance on the content and the ownership of the R\&M 4 case through the life of a system, and advocate the creation of an "Initial R\&M Case Report" in order to capture fully the AR\&M requirements and areas of AR\&M related risks.

The SAM modelling activities discussed above support the development of such an Initial R\&M Case Report during the concept and assessment phases, by:

- $\quad$ Providing full traceability of requirements, through documentation of the SAM model input data (e.g. Mission profiles, major functions, equipment AR\&M data and sources), assumptions and stakeholder decisions;

- Demonstrating the link between equipment end-user and System Requirements, by documenting the modelling approach undertaken to generate the requirements; and

\footnotetext{
${ }^{2}$ Typically, the variate is expressed as time (i.e. MTBF) or miles.

${ }^{3}$ Cost of ownership can be determined by analysing outputs from SAM, rather than directly from a SAM model.

4 Note, BS EN 62741 [2] refers to dependability case, rather than R\&M case, but is in essence the same for the purposes of this paper.
} 
- Aiding in the identification of areas of risk, through sensitivity analysis as described in the sections above.

\section{Managing Risk with SAM}

\subsection{Progressive Assurance}

As quoted in Part B of GR-77 [2]:

"AR\&M is built into a system at the design stage. No amount of analysis or testing can improve a design without a costly revisit to the design stage. The use of good design practice for high reliability and good field maintainability (in software and hardware) is an essential component of reducing project risk and achieving good products."

It is therefore essential to initiate the AR\&M programme early in a project design/development phase.

Assurance that a developed solution satisfies the defined AR\&M requirements is generally the responsibility of the contractor, where any modelling activities may be performed using commercially available or bespoke modelling tools (for example, RAM4, which is also free to use for MOD projects, or SAM).

These assurance activities should be initiated as early as possible in order to influence design activities, and identify areas of risk and opportunities for improvement. Progressive assurance is intended to provide the contracting project/delivery team with regular updates of predicted performance, areas of risk and potential for trade-off.

By maintaining and updating the SAM model developed to set the requirements, the project/delivery team can use the information provided by the contractor to:

- Investigate the impact of predicted performance and risk areas on fleet level metrics and the equipment end-user requirements;

- Assess design options and perform trade off studies against the fleet level requirements.

Throughout the project design/development phase, the way in which the equipment or fleet is to be used may be subject to changes driven by the equipment end-user, the project/delivery team, and/or the contractor's engineered solution, such as:

- Changes in how and where the equipment or fleet is to be operated may affect the mission profile, usage and environment.

- Changes in how the equipment or fleet is supported may affect spares holdings, logistic delay times and as such equipment repair times. This may also ultimately affect the mission profile of a fleet, if it cannot be supported appropriately.

Reflecting these changes in the SAM model, as they arise, allows the project/delivery team to assess the impact on the fleet performance and AR\&M requirements. It also enables them to understand the drivers of fleet unavailability/unreliability, for example specific mission activities, equipment and/or their functions. Therefore, through regular review of the AR\&M development progress, the project/delivery team can better understand and manage the risks associated with achieving the system and equipment end-user requirements throughout the project design/development phase. This is key to the successful delivery of a project.

\subsection{Assessing System or Function Significance}

The development of a criticality matrix to create a SAM model, as described in Section 3.2.1, allows the significance of individual functions or sub-systems to be analysed and therefore used in the management of project risk.

Varying of the model input parameters (reliability and maintainability data, or details of the mission profiles), through sensitivity analysis, can be used to identify those functions and hence sub-systems or equipment which have the greatest impact on overall fleet performance or the performance of critical functions (e.g. safety critical activities such as replenishment at sea or close water navigation). As such, those model input parameters, which have the greatest impact on performance, can then be the focus of assurance activities (as described above) as part of a risk-based approach.

\subsection{Validating Spares Recommendations}

Many projects require the contractor to provide a list of recommended spares to support their equipment during a pre-defined mission duration. These lists typically comprise three elements:

- The list of consumable items, which can be estimated from contractor and equipment end-user experience of attrition rates in service; 
- The list of spares required to support scheduled maintenance activities, which can be developed from the details of each maintenance activity.

- The list of spares required to support corrective maintenance activities when components fail. Deciding which spares to take on a mission, to cover component failures, should be based on an understanding of the risk to mission success. Spares modelling tools, used to provide these recommendations, typically take account of system architecture, equipment reliability, and cost of spares to calculate the most cost effective solution to meet a target metric (e.g. operational availability of a system) over the mission duration. However, these tools may not be able to reflect a complex mission profile for a fleet of assets, where different equipment functions are required for different phases of the mission, and in particular, where the system architecture (required for mission success) may change over time; this is where SAM can be used to validate any spares recommendation.

It is important to note that SAM is not designed to provide a spares recommendation; instead, as discussed in Appendix A (Section 6), the MEL includes the quantity of spares supporting each item modelled. By including the list of recommended spares into the developed SAM model, undertaking sensitivity analysis, and interrogating the SAM outputs, it is possible to validate the spares recommendation against achieving the mission profile. This can include identifying any shortfalls in sparing, or where the number of spares has a lesser impact on mission success (for example, because a system function is only required for short period of the mission).

The results from the SAM model can then be used to influence the decision on type, quantity and location of spares required to support a mission. Moreover, as mission requirements change, the SAM model can be adapted accordingly to allow the project/delivery team to understand the impact on sparing requirements.

Finally, feeding experience from in-service back into the models (spares model and SAM) is key to validating the approach and providing better estimations in the future.

\subsection{Supporting System Acceptance}

The concept of a digital twin can be defined as a digital replica of physical assets, which can be used to optimise the operation and maintenance of physical assets or systems. In this way, the modelling approach described in this paper can act as a digital twin to a fleet of assets in relation to their AR\&M performance. Clearly, the effectiveness of a digital twin is reliant on it reflecting reality; for an AR\&M model, this includes incorporating in-service reliability and maintainability performance, and reflecting the system design, usage and mission profile.

At the end of a project design/development phase, the SAM model should reflect the detailed design, incorporate results from any reliability trials/tests, and importantly the AR\&M-related risks should be well understood. Throughout the manufacturing/installation phase, the SAM model should be updated to reflect the final as-built designs, incorporating any design changes occurring during manufacture/installation. Furthermore, any defects or issues identified during manufacturing, which counter the claims presented in the R\&M case report should be documented and reflected, as appropriate, into the SAM model.

The purpose of this is to provide input into the system acceptance activities to demonstrate that the system satisfies all AR\&M requirements documented within the SRD and URD. As discussed in Section 3, the SAM model can be used to develop the project requirements; as such, the same "as-built" SAM model can be used to verify and validate the requirements, and support formal acceptance of the R\&M Case.

Part of the verification and validation activity may include the collection of in-service performance data. In this case, the use of the SAM model allied to a rigorous approach to recording and sentencing data, (e.g. through a formal Data Reporting, Analysis and Corrective Action System (DRACAS)) should be planned for the early in service phase. This will allow the programme to focus upon reliability growth and the availability requirements rather than an artificial "demonstration" which places certain constraints, such as fixed build standard, upon the equipment supplier and the customer. The approach should employ suitable modelling tools, such as SAM, in order to ensure that a route to acceptance of the system, will actively involve both the project/delivery team and the equipment supplier at an early stage. The approach minimises the potential for dispute and ambiguity when dealing with the often-contentious issue of proving AR\&M characteristics. Critically the method allows for a changing build standard, minimises the duration of the proving period and promotes a partnering approach to acceptance of the system AR\&M characteristics.

The SAM model, reflecting the manufactured solution, can then be used to provide continued assurance, act as a digital twin, and support decision making during the in-service phase.

\section{5. $\quad$ Reflecting Actual In-Service Performance}

The primary reason for updating and maintaining the "as-built" SAM model during the in-service phase, is to incorporate in-service reliability and maintainability data, and reflect actual usage, to provide continued assurance against the equipment end-user requirements and specified project KPIs. 
In addition to this, the SAM model can be used to:

- Assess the impact of proposed changes to system design, usage, and support structure, on the AR\&M performance.

- Influence the development of AR\&M requirements for new projects, by reporting on equipment, system and fleet level metrics.

- Aid in decision making to drive down cost of ownership. Although cost information is not directly included in the SAM model, the cost of ownership of a fleet of assets is dependent on a number of the factors represented within the SAM model, such as equipment/asset reliability, maintenance times, and usage and mission parameters. Through interrogation of the in-service SAM model, and combining these factors with actual costs it is possible for project/delivery teams to identify how the factors are influencing the total cost. This information can then be used to support options studies seeking to drive down the cost of ownership, and understanding the impact on fleet and asset AR\&M performance: a key parameter to be considered in all options studies.

The $\mathrm{R} \& \mathrm{M}$ case is employed to provide progressive assurance of compliance against the AR\&M requirements, and where not, a reasoned auditable argument is presented as to why not. The R\&M case is developed through a risk-based approach.

Actual AR\&M performance of a system is unknown (i.e. the risk that the system will not achieve its AR\&M requirements is greatest) during the early phases of a project (concept/assessment and development phases). Modelling activities are used to predict the future performance of the system in order to reduce the risk to the project.

When the project enters the in-service phase, AR\&M data should be collected and the actual in-service performance of the system incorporated into the "as-built" SAM model to assess performance against the AR\&M requirements. However, the reliance on this data during the early in-services stages of the project, to provide assurance of the AR\&M performance, should be limited due to the following reasons:

- The sample size of data from which to draw conclusions is initially small therefore there is uncertainty in the early data.

- The systems may be subject to early life failures as defective products are identified and discarded, and early sources of potential failure are surmounted.

- Operating and maintenance procedures may still be being established and honed (which may affect the AR\&M performance of the system).

Initial in-service data should be used to identify areas for improvement within the design, and the predicted performance (from modelling activities) should be used to provide assurance. The statistical significance of the in-service data increases with the amount of time in service and the quantity of data collected, and the reliance on the predicted performance to provide assurance therefore decreases. This is illustrated in Figure 5.

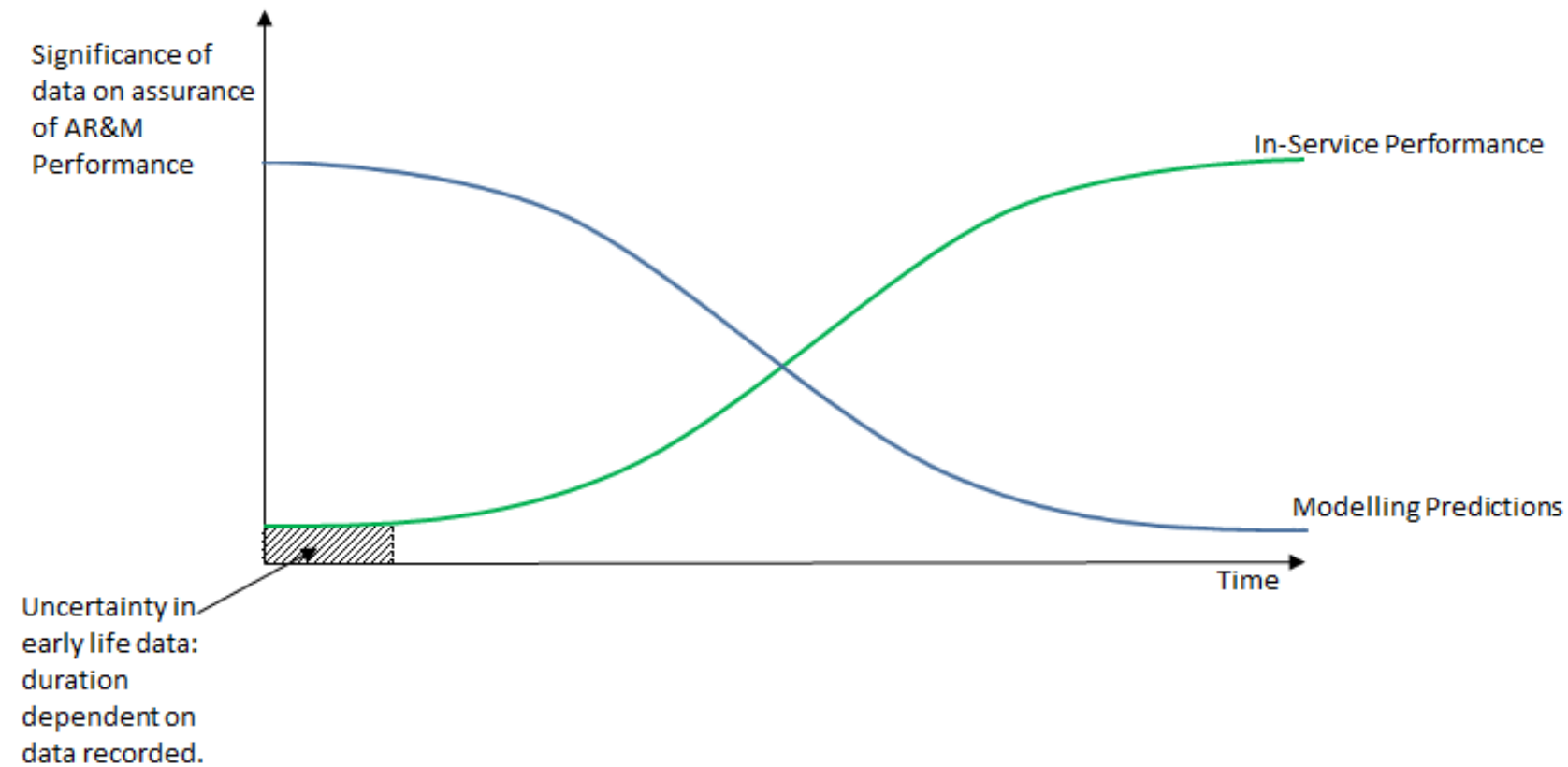

Figure 5: Significance of In-Service Performance and Modelling Predictions over Time 


\section{Concluding Remarks}

The British statistician, George E. P. Box famously wrote, in various books and papers, "All models are wrong, but some are useful". This has been borne out from my experience of over 12 years' modelling complex systems and managing AR\&M software tools. As such, I felt it important to highlight guidance and tools that exists to provide valuable and insightful modelling, that helps set realistic requirements, and can be used to understand the risks of operating complex fleets of assets in service.

The modelling approach discussed within this paper can be employed as a manually updated digital twin to a fleet of assets, reflecting in-service performance. While it is unlikely that complex availability models can be automatically updated with reliability data in the short term, these models can (and should) be periodically reviewed and updated from in-service experience. This allows the models to be used to analyse the effect of changes in design and usage upon fleet level AR\&M metrics and KPIs, before they are implemented - hence saving time and money.

\section{References}

1. Applied R\&M Manual for Defence Systems, Part B - R\&M Related Activities, Chapter 2 - R\&M Performance Specification, Version 1.

2. Applied R\&M Manual for Defence Systems, Part B - R\&M Related Activities, Version 1.

3. BSI Standards Publication, BS EN 62741, Demonstration of dependability requirements - The dependability case, 2015.

4. Ministry of Defence, Defence Standard 00-42, Reliability and Maintainability Assurance Case, Part 3: R\&M Case, Issue 5, May 2016.

\section{Glossary of Terms}

\begin{tabular}{|l|l|}
\hline \multicolumn{1}{|c|}{ Acronym } & \multicolumn{1}{c|}{ Definition } \\
\hline ADC & Availability Dependency Chart \\
\hline AR\&M & Availability, Reliability and Maintainability \\
\hline ART & Active Repair Time \\
\hline Def Stan & Defence Standard \\
\hline DRACAS & Data Reporting, Analysis and Corrective Action System \\
\hline KPI & Key Performance Indicators \\
\hline MART & Mean Active Repair Time \\
\hline MEL & Model Equipment List \\
\hline MOD & Ministry Of Defence \\
\hline MPC & Mission Profile Chart \\
\hline MTBF & Mean Time Between Failure \\
\hline MTTR & Mean Time To Repair \\
\hline R\&M & Reliability and Maintainability \\
\hline SAM & Systems Availability Model \\
\hline SaRS & Safety and Reliability Society \\
\hline SRD & System Requirements Document \\
\hline URD & User Requirements Document \\
\hline
\end{tabular}




\section{Appendix A - SAM Details}

\subsection{General}

As previously stated, SAM is a program designed to assess the AR\&M characteristics of multiple systems used over operating scenarios that place varying demands upon those systems, such as that encountered in complex military, industrial installations and deployed systems or systems.

The flexibility of the program results from the assessment method utilised, which employs Monte Carlo simulation techniques. The system to be modelled is defined using three input screens known as "Charts":

1. Model Equipment List (MEL)

The MEL breaks down equipments into component data (also, see note below). Component characteristics are defined by how often they fail (e.g. MTBF), and Equipment characteristics include repair and sparing requirements (e.g. Mean Active Repair Time (MART), and the probability that the component can be repaired during the mission).

2. Availability Dependency Chart (ADC)

The ADC assembles equipments (defined in terms of failure and repair data, which has been either transferred from the MEL or entered directly into the ADC) into major functions using dependency logic to allow for redundancy, where appropriate. An example a major function may be to "Provide Maximum Power", which utilises 3 from 3 diesel generators.

3. Mission Profile Chart (MPC)

The MPC overlays the system dependency (in terms of major functions defined in the ADC) onto a set of activity types (e.g. ship transit) using a criticality matrix. This allows the combination of major functions to be defined for each activity type (e.g. the activity type "Ship Transit" requires the major function "Maximum Power"). Missions are then developed as a sequence of activities (made up from defined activity types) each lasting for a specific period. Failure of an activity results in the model entering a "Fallback Activity", which is also defined in the MPC.

Note, although described in terms of "equipment" and "components", the SAM model may be defined in terms of "system" and "sub-systems", or any other combination of higher and lower level items deemed appropriate for the project stage and modelling purpose.

SAM performs rigorous data checks following the completion of each chart, and provides the SAM user with details of any errors that have been identified. Six types of output option are provided from the SAM simulation:

1. Input Data

Allows the chart data used to build the model to be examined.

2. Mission Definition

Allows the input parameters to the simulation to be examined.

3. Development Data

Allows data for each individual mission in the simulation to be examined.

4. Logistics

Used for investigating the allocation and effects of spares and repairs on the availability of a system, such as the number of failures, and spares required for particular equipments.

5. Reliability

Used for investigating the reliability of the system, major functions and activities. Also used to examine the end states for equipment and major functions.

6. Availability (only used for availability models - see note below)

Used for investigating the availability of the system and to determine the major function unavailability drivers. This output also examines the end states for equipment and major functions and outputs the instantaneous availability levels at mission end for selected functionality in the ADC.

Note: There are two types of SAM models:

a. Reliability models in which missions are terminated upon failure

b. Availability models in which fallback activities are used to monitor downtime in order to measure uptime and downtime over a mission period.

The process for creating and running a SAM model, as discussed above, is presented in Figure 6. 


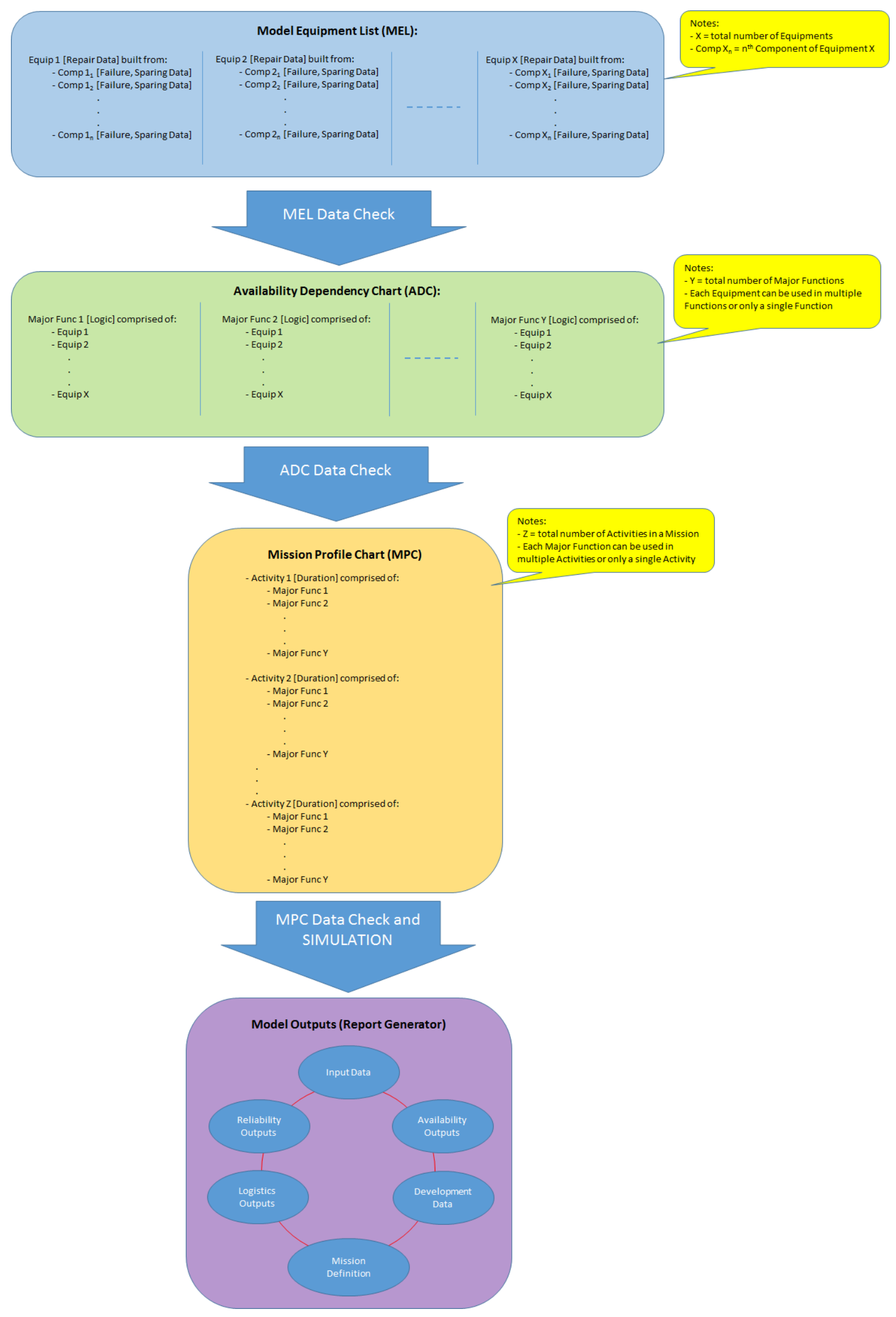

Figure 6: SAM Model Construction 